\title{
EXTENSIONS OF FATOU'S INEQUALITY
}

\author{
LIVIU C. FLORESCU
}

Abstract. Using some compacity techniques in the space of integrable functions we obtain an expression of the gap in the Fatou's inequality. Also, we derived as corollaries some results of H.-A. Klei.

Mathematics subject classification (2000): 28A20, 46E30.

Key words and phrases: Young measures, biting lemma, modulus of uniform integrability.

\section{REFERENCES}

[1] Brooks, J. K. And Chacon, R. V., Continuity and compactness of measures, Adv. Math., 37 (1980), 16-26.

[2] FloResCU, L. C., $w^{2}$ - Pseudouniformities and biting lemma, Boll. Un. Mat. Ital. A (7) 7-A (1993), 87-96.

[3] FloresCU, L. C., Weak compactness results on $L^{1}$, An. Ştiinţ. Univ. Al. I. Cuza Iaşi Mat. (N.S.), t.XLV, f.1 (1999), 75-86.

[4] Florescu, L. C. ET Godet-ThobIe, C., Quelques propriétés des mesures de Young, An. Ştiinţ. Univ. Al. I. Cuza Iaşi Mat. (N.S.), t.XLVI, f.2 (2000), 393-412.

[5] KLEI, H.-A., Measure convergent sequences in Lebesgue spaces, Bull. Austral. Math. Soc. 54 (1996), 197-202.

[6] KLEI, H.-A., Fatou's identity andLebesgue's convergence theorem, Proc. A.M.S. 127 (1999), $2297-2302$.

[7] Rosenthal, H. P., Sous-espaces de $L^{1}$, Lectures Univ. Paris VI (1979).

[8] SaAdoune, M. AND VAladier, M., Extraction of a "good" subsequence from a bounded sequence of integrable functions, J. Convex Anal. 2 (1995), 345-357.

[9] VALADIER, M., A course on Young measures, Rend. Istit. Mat. Univ. Trieste, XXVI (1994), Supplemento, 349-394. 